\title{
Assessing cue usage in performance contexts: A comparison between eye-movement and concurrent verbal report methods
}

\author{
A. M. WILLIAMS \\ Liverpool John Moores University, Liverpool, England \\ and \\ K. DAVIDS \\ Manchester Metropolitan University, Stoke-on-Trent, England
}

\begin{abstract}
The relationship between eye fixations and concurrent verbal reports as measures of selective attention was examined in two different performance contexts. Experienced and less experienced soccer players were presented with filmed action sequences. In Experiment 1, subjects reacted to 11versus-11 soccer simulations; in Experiment 2, 3-versus-3 situations were presented. Each study involved two experimental conditions: (1) visual fixations were recorded using an eye-movement system and (2) subjects continuously verbalized the location of their visual attention. In 11-versus11 situations, there were no differences in performance across the two conditions, indicating that verbalization had no effect on performance. Also, there were no differences between the two methods in identifying important information sources. In 3-versus-3 situations, concurrent verbalization resulted in reactivity. Moreover, there were differences between the two methods in identifying important display areas. Findings showed that the relationship between visual fixation and selective attention is dependent on the nature of the stimulus presented. When peripheral vision is employed to extract task-specific information, verbal reports provide a more veritable measure of selective attention; eye fixations may yield a more accurate measure when the task requires information to be extracted foveally. Findings are discussed with regard to the differences between "looking" and "seeing" as a methodological issue.
\end{abstract}

Human performance research examining proficiencyrelated differences in visual search strategy has assumed that eye-movement data and interspersed fixations are indicative of the perceptual approach used by the performer to extract meaningful information from the display (e.g., Abernethy, 1990; Hella, 1984; Papin, 1984; Zegers, 1986; for a review, see Abernethy, 1988). That is, visual fixation characteristics are taken as reflecting the strategy used by the observer to extract task-specific information. Fixation location is assumed to reflect the important cues used in decision making, whereas the number and duration of fixations are presumed to indicate the information-processing demands on the individual (Abernethy, 1985).

These assumptions have been subject to a number of criticisms. First, visual orientation, as implied from fixation characteristics, may not be directly related to visual attention and selective information pickup (see Abernethy, 1988; Davids, 1984; Williams, Davids, Burwitz, \& Williams, 1993). It appears that target objects in the en-

This study was completed as part of the first author's doctoral degree at the University of Liverpool. Correspondence should be addressed to A. M. Williams, Centre for Sport and Exercise Science, School of Human Sciences, Liverpool John Moores University, Byrom Street, Liverpool L3 3AF, England (e-mail: m.williams@livjm.ac.uk). vironment can be fixated without extracting specific information (e.g., Kundel, Nodine, \& Toto, 1984; Papin, Metges, \& Amalberti, 1984; Stager \& Angus, 1978). This has been referred to as the difference between "looking," which implies fixation on the fovea, and "seeing," which intimates information processing or cue extraction (Abernethy, 1988; Neumann, van der Heijden, \& Allport, 1986; van der Heijden, 1986). Second, it seems that attention can be moved around the visual field without making distinctive eye movements to change the point of fixation (see Jonides, 1981; Remington, 1980; Sanders \& Houtmans, 1985; Shulman, Remington, \& McLean, 1979). That is, subjects may "look" at, or foveate, an object but extract information from the periphery. For example, a car driver may fixate on the road directly in front of the vehicle while picking up information from peripheral vision regarding the orientation of the car relative to the curb or other vehicles. These shifts in attention can also occur without the loss of input information normally associated with saccadic eye movements (Abernethy, 1985). This may result in subjects' giving priority to information from the peripheral visual field when appropriate (Abernethy, 1988). In fact, a growing body of evidence suggests that information processed through peripheral vision plays an important role in perceptuo-motor performance (e.g., Davids, 1984; Paillard, 1982; Williams, Davids, 
Burwitz, \& Williams, 1992). Perhaps fixations simply provide the most appropriate reference points for picking up and organizing information from the periphery (Ripoll, 1991; Rockwell, 1972).

The problems involved when relying solely on eyemovement registration techniques to provide information on selective information pickup have recently been highlighted by Williams, Davids, and Burwitz (1995a) using a popular team game as the vehicle. In this study, the visual search strategies of experienced and less experienced soccer players were recorded while they viewed 3 -versus-3 action sequences. Although the experienced players responded quicker and more accurately to the film task, it is important to note that no differences were observed between groups in the allocation of fixations to selected areas of the display. Specifically, both groups of subjects fixated primarily on the ball or the player in possession of the ball. However, in a related study, Williams, Davids, and Burwitz (1995b) used a spatial or event occlusion approach to demonstrate that the experienced players were able to more effectively use peripheral vision to extract task-specific information in these situations. They found that when they masked information pickup from areas other than the ball or player in possession of the ball, it affected the experienced players' performance more than it affected the less experienced players' performance. Since there were no differences between groups in foveal orientation, these data support the view that the experienced players extracted more information through nonfoveal stimulation (i.e., from the parafovea and periphery). This research clearly highlights the need to combine eye-movement registration techniques with parallel measures of information extraction when attempting to isolate the perceptual strategies employed by performers.

Verbal report procedures require subjects to verbalize the area of the display that they consider to be particularly informative. Consequently, a more direct measure of attentional allocation and information extraction is provided (Bainbridge, 1990; Ericsson \& Simon, 1980, 1993). Data can be collected either during performance (i.e., concurrently) or retrospectively following completion of the task (Green, 1995). However, despite these advantages, few studies have used verbal protocol analysis to examine visual attention during performance. Furthermore, there have been even fewer attempts to validate eye fixations with supporting information based on verbal reports.

Williams and Burwitz (1993) used a retrospective technique to identify cue usage by soccer goalkeepers during a penalty kick. The results showed that the primary sources of information used by experienced goalkeepers included the position of the penalty-taker's hip and the angle of the trunk and foot prior to ball contact. Interestingly, these data concurred with eye-movement data obtained in an earlier study by Tyldesley, Bootsma, and Bomhoff (1982), suggesting a positive relationship between verbal reports and eye fixations. A retrospective approach has also been used in other sport contexts. For example, Buckolz, Prapavesis, and Fairs (1988) examined cue usage in filmed tennis simulations by requiring subjects to indicate the areas of the display that were most informative in anticipating the direction of their opponents strokes. Several important visual cues were successfully identified from the verbal report data.

Similarly, verbal reports have been used to investigate search strategy and cognitive processing in tasks such as driving a car or landing a helicopter (Berbaum, Kennedy, \& Hettinger, 1991; Hughes \& Cole, 1986). Hughes and Cole required subjects to continuously report the areas of the display that attracted their attention while driving. Findings showed that drivers devoted a good proportion of their attention to other vehicles, pedestrians, and advertising when driving through a crowded shopping center. However, considerably less attention was given to traffic control devices and other surrounding information such as road surface markings and layout. Verbal protocols have also been used to report mental processes in tasks such as computer hardware diagnosis (Reed \& Johnson, 1993), computer programming (Koubek \& Salvendy, 1991), and computer interface design (Jorgensen, 1990). These studies suggest that verbal reports can be a valid and reliable measure of cognitive processing during performance (Green, 1995).

However, there is also evidence to suggest that verbal reports may be ineffective in identifying cognitive processing during task performance (see Le Plat \& Hoc, 1981 ; Nisbett \& Wilson, 1977). Nisbett and Wilson argue against the possibility of accessing cognitive processes via verbal report protocols. The suggestion is that verbal reports may be contaminated by subject biases and a priori expectations and may be no more accurate than a rational reconstruction of events from a third-person perspective. That is, retrospective reports can be corrupted by the subject's attempts to "tidy up" what actually happened or to rationalize what occurred (Green, 1995). Furthermore, some studies have indicated that verbal reports suffer from reactivity (e.g., McGeorge \& Burton, 1989; Russo, Johnson, \& Stephens, 1989). Reactivity occurs when the normal function of cognitive processes is disrupted by requiring subjects to verbally report during the experiment. That is, verbalization may change the normal way that a task is performed in exchange for an approach that is easier to describe. Similarly, on certain tasks, verbalization may increase the time required to perform the task. Alternatively, the speed of the ongoing processes may surpass the ability of the subject to verbalize them completely (Brinkman, 1993).

Nonetheless, most cognitive psychologists agree that, if used appropriately, verbal reports can provide valuable insights into higher mental processes (Brinkman, 1993; Ericsson \& Simon, 1980; Green, 1995). Ericsson and Simon (1993) suggest that verbal reports are valid, providing that certain principles are adhered to in the experimental procedure. They argue that, wherever possible, concurrent verbal reports should be collected and analyzed. This procedure enables subjects to verbalize the information they are currently processing in short-term 
memory (Ericsson \& Oliver, 1989). With retrospective reports, the information may no longer be available in short-term memory but has to be retrieved from longterm memory. Since retrieval from long-term memory is fallible, this can result in important sources of information being omitted or redundant information being included (e.g., see Brinkman, 1993; Russo et al., 1989). Furthermore, with retrospective reports, it is difficult to distinguish information attended to during the task from information acquired or used after (or even before) the completion of the task (Green, 1995).

The purpose of this research was to examine how experienced and less experienced performers in a team sport such as soccer differ in their selection of information and to investigate whether this question can be reliably investigated with verbal protocols and eye-movement recording methods. Also, the research examined whether experience and the type of task have a mediating effect in determining the efficacy of either method as a measure of selective attention. Specifically, the experiments reported here intended to determine the relationship between subjects' locus of attention and their locus of fixation during performance on two different film-based anticipation tasks. Since verbal reports provide a direct measure of attentional allocation, the aim was to examine the association between visual orientation (as implied from eye-fixation data) and visual attention. If a meaningful relationship were demonstrated, this would support the validity of using either method as a measure of selective attention in human performance research.

\section{EXPERIMENT 1}

Experiment 1 examined the relationship between visual fixation and selective attention during 11 -versus-11 soccer simulations. Previous research has demonstrated that subjects should be aware of the positions and movements of players "off the ball" when attempting to anticipate pass destination in these circumstances (Williams, Davids, Burwitz, \& Williams, 1994). To achieve this, players need to adopt an extensive search strategy involving numerous fixations of short duration (see Williams et al., 1994). Such a search strategy enables them to utilize foveal vision to pickup important information from the positions and movements of players, rather than relying on less precise information through nonfoveal stimulation. Consequently, because of the importance of information pickup through foveal or central vision, it was hypothesized that there would be a positive relationship between verbal reports and eye fixations in these 11 -versus-11 simulations.

\section{Method}

\section{Subjects}

The subjects were 10 experienced male soccer players $(M$ age $=$ 20.8 years, $S D=1.5$ ) and 10 less experienced male soccer players ( $M$ age $=20.6$ years, $S D=2.1$ ). The experienced players had been playing soccer for 12.4 years $(S D=2.1)$ and had played an average of $620(S D=180)$ competitive matches. They included 6 uni- versity first-team players and 4 semiprofessional players. The less experienced group contained recreational and university thirdteam players with 5.6 years playing experience $(S D=2.5)$, who had participated in an average of $85(S D=55)$ competitive matches. The subjects gave their informed consent to participate in the experiment.

\section{Test Film}

The subjects were presented with a total of 26 soccer action sequences. These sequences were selected from a sample of 14 university and professional soccer matches filmed over a 2-month period. The games were filmed from a position behind $(15 \mathrm{~m})$ and slightly above $(5 \mathrm{~m})$ the goal using a video camera, an $8-\mathrm{mm}$ focal length lens, and a video recorder. This position enabled the entire width of the playing field to be viewed on film. Prior to filming the soccer matches, a response reference grid was painted on the field of play using yellow marker. This provided 10 reference grids, numbered 1 to 10 , which were used in measuring subject responses. The arrangement of the 10 response grids is highlighted in Figure 1.

Each clip of film lasted approximately $10 \mathrm{sec}$ and included an offensive pattern of play, ending with a pass into one of the 10 reference grids. The player making the final pass was surrounded by a black reference square during the entire trial so that he could be clearly highlighted. This enabled passing sequences to evolve as normal on screen, with subjects' only having to anticipate the pass made by the highlighted player. The reference square subtended a visual angle of approximately $6^{\circ}$ in the horizontal direction and $5^{\circ}$ in the vertical direction. Care was taken to ensure that the square did not mask the player's actions on screen. An intertrial interval of $8 \mathrm{sec}$ was built into the film construction.

\section{Apparatus}

An Applied Science Laboratories (ASL) 4000SU eye-movement registration system was used to collect visual search data. This is a video-based monocular system that measures eye line of gaze with respect to a helmet-mounted scene camera. The system works by detecting two features, the pupil and the corneal reflex (reflection of a light source from the surface of the cornea), in a video image of the eye. The relative position of these features is used to compute visual gaze with respect to the optics. Displacement data from the left pupil and cornea were recorded by a small camera,

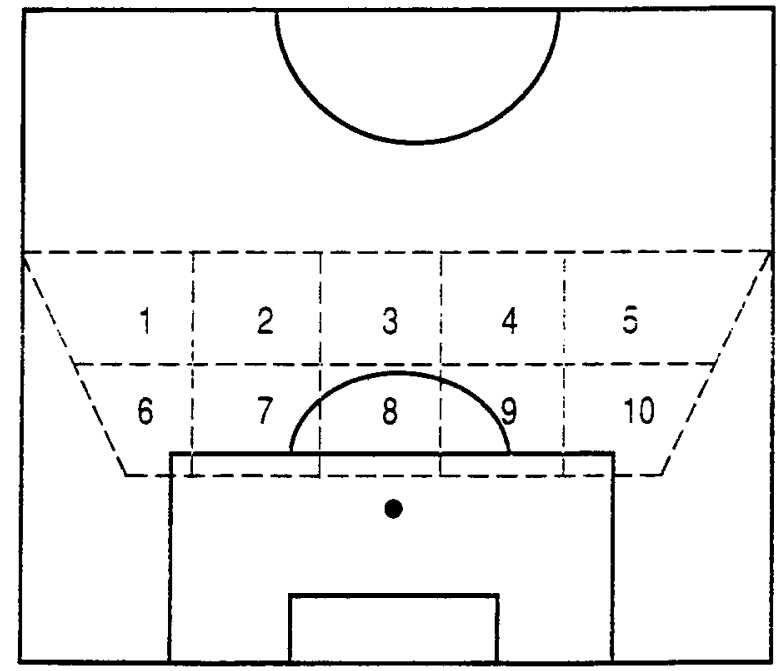

Figure 1. Diagram showing the reference grids marked on the field of play. 
processed by computer, and superimposed as a cursor on the scene camera image to highlight the point of gaze. This image was then recorded using a video recorder for further analysis. The data were then subjected to a frame-by-frame analysis using a UK standard video recorder at 50 frames per second. The accuracy of the system (i.e., the difference between true eye position and measured eye position) was $\pm 1^{\circ}$ visual angle. System precision (i.e., the amount of instrument noise in the eye-position measure when the eye is perfectly stationary) was better than $0.5^{\circ}$ in both horizontal and vertical directions (see Applied Science Laboratories, 1992, for a more detailed review of the apparatus ${ }^{1}$ ).

\section{Procedure}

Eye-movement condition. Prior to presentation of the test film, the subjects' choice reaction time (CRT) was measured. An empty soccer field containing the 10 reference grids and their corresponding numbers was presented as the display. Following a random foreperiod, the subjects were required to respond as quickly and as accurately as possible when a stimulus was presented. The stimulus was a black reference square that surrounded 1 of the 10 grid numbers. The subject's task was to verbally indicate the number inside the square. The subjects were presented with 3 practice trials and 22 test trials. CRT was measured as the time from the presentation of the stimulus to the subject's verbal response, recorded using a series of timing circuits, a voice reaction timer, and a computer. The CRT task served to familiarize the subjects with the response required in the anticipation test and provided the baseline measure of CRT used as a covariate in the subsequent performance analysis.

The test procedure was then explained and the helmet-mounted optics were fitted on the subject's head. The eye-movement recorder was calibrated using a 9-point reference grid so that the recorded indication of fixation position corresponded to the subject's visual gaze. Previous research (e.g., Williams et al., 1994) showed that the system required only occasional recalibration, needing to be checked only after the practice trials and following presentation of the first 13 test trials. Also, the subjects were asked to fixate on a marker positioned in the center of the projected film image immediately after completing their verbal response, thus enabling a rapid calibration check prior to each film trial. All subjects reported that the helmet was comfortable and did not interfere with performance.

Following the initial calibration, the subjects received 12 practice trials to ensure that they were familiar with the testing procedure. In particular, it was stressed that they should anticipate pass destination as quickly and as accurately as possible. No further instructions were given, in order to avoid modifying search strategy by experimental instructions (Yarbus, 1967). The test film was then presented using a video projection system and a $3.5 \times 3 \mathrm{~m}$ screen. The subjects sat $5 \mathrm{~m}$ away from the screen so that the film image subtended a visual angle of approximately $40^{\circ}$ in the horizontal direction and $35^{\circ}$ in the vertical direction. The subjects viewed each pattern of play as it developed on screen. When the ball was passed to the player highlighted inside the black square, the subjects were required to verbalize as quickly and accurately as possible the grid number anticipated as the final pass destination (see Figure 1). Immediately after their verbal response, the film was occluded to prevent the subjects from gaining feedback on task performance. The time from the start of the film sequence to the subject's response was recorded using a voice reaction timer and microcomputer. After the first 13 film trials, the subjects were given a 2 -min rest period during which the eye-movement system was recalibrated. The entire test session, including practice and calibration, lasted approximately $30 \mathrm{~min}$.

Verbal report condition. First, the subjects' CRT was measured using the same test film as in the eye-movement condition. In this CRT test, the subjects were required to respond by pressing a button to stop a reaction timer while simultaneously verbalizing the appropriate box number. The time from the start of the film sequence to the subject's manual response was recorded using the same reaction timer and computer. The CRT task served to familiarize the subjects with the response required in the anticipation test and provided the baseline measure of CRT used as a covariate in the subsequent performance analysis. Also, differences in the subjects' CRT values, when using verbal response measures compared with manual response measures, were used to control for any confounds that may have arisen when comparing performance in the eye-movement and verbal report conditions.

In the verbal report condition, the subjects viewed the same test film as in the eye-movement condition. However, the sequence of presentation of film clips was changed to minimize order effects. Also, presentation of the conditions was counterbalanced to control for possible learning biases. A test procedure similar to that in the eye-movement condition was adopted. The subjects viewed each pattern of play as it developed on screen. When the ball was passed to the player highlighted inside the black square, the subjects were required to indicate as quickly and accurately as possible the box number anticipated as the final pass destination. The subjects responded by pressing a response button, to stop a reaction timer, while verbally indicating the box number intended as final ball destination. Immediately after responding, the film was electonically occluded to prevent the subjects from gaining feedback on task performance. It was stressed that the subjects should respond as quickly and as accurately as possible to the filmed stimuli.

As each pattern of play unfolded on screen, the subjects were required to continuously indicate the area of the screen from which they were extracting information. That is, they were asked to verbalize the area of the screen to which they were allocating attention at that specific moment in time. To simplify this process, and to minimize possible reactivity effects, the screen was divided into three main areas: (1) box, the black reference square that highlighted the player who would make the eventual pass and, for a large portion of the trial, the ball; (2) right, the right side of the screen; and (3) left, the left side of the screen. The subjects' verbalizations were recorded using a video camera and recorder and were later transcribed verbatim for analysis. The camera was positioned to simultaneously record the subjects' verbal comments and the film image displayed on the screen. This enabled the subjects' verbal reports to be coupled with the filmed action sequences for further analysis. The subjects then received 12 practice trials to ensure familiarity with the verbalization procedure. In addition, trials in which there was a breakdown in verbalization processes were discarded. Since the subjects found the verbalization procedure to be uncomplicated, few trials were actually rejected. The entire test session lasted for $30 \mathrm{~min}$ on average.

\section{Dependent Variables and Analysis for the 11-Versus-11 Anticipation Test}

The reactivity of the verbal report technique was tested by comparing performance on the anticipation test in the verbal report condition with the same data from the eye-movement condition. The following dependent measures were obtained:

Response time (RT). The RT was defined as the time (in milliseconds) from the start of the film clip to the subject's verbal response (eye-movement condition) or manual response (verbal report condition).

Verbal response error (VRE). The VRE was obtained by comparing the subjects' verbal response to the actual final ball destination (in percentages).

In addition to these dependent variables, two covariates were included in the statistical analysis. These were the CRT values obtained using the verbal response method in the eye-movement condition (VCRT) and the manual response procedure in the verbal report condition (MCRT). The purpose of using the CRT values as covariates was to control for any confounds that may have arisen as a result of using different response modes in the two conditions. ${ }^{2}$ 
Table 1

Differences in Anticipatory Performance in the 11-Versus-11 Situations Between Experienced and Less Experienced Soccer Players Across the Eye-Movement and Verbal Report Conditions

\begin{tabular}{|c|c|c|c|c|c|c|c|c|c|c|c|c|}
\hline \multirow[b]{3}{*}{ Group } & \multicolumn{6}{|c|}{ Eye-Movement Condition } & \multicolumn{6}{|c|}{ Verbal Report Condition } \\
\hline & \multicolumn{2}{|c|}{ VCRT (msec) } & \multicolumn{2}{|c|}{ VRE (\%) } & \multicolumn{2}{|c|}{ RT (msec) } & \multicolumn{2}{|c|}{ MCRT (msec) } & \multicolumn{2}{|c|}{ VRE $(\%)$} & \multicolumn{2}{|c|}{ RT (msec) } \\
\hline & $M$ & $S D$ & $M$ & $S D$ & $M$ & $S D$ & $M$ & $S D$ & $M$ & $S D$ & $M$ & $\underline{S D}$ \\
\hline & 472 & 103 & 3.7 & 7.0 & 9 & 2 & 353 & 62 & 18.6 & 12.0 & 9,144 & 355 \\
\hline Less experienced $(n=10)$ & 450 & 79.3 & 16.7 & 11.0 & 9,623 & 413.2 & 343 & 49 & 17.0 & 9.3 & 9,676 & 320 \\
\hline
\end{tabular}

Note-VCRT = verbal response choice reaction time; $\mathrm{VRE}=$ verbal response error; $\mathrm{RT}=$ response time; $\mathrm{MCRT}=$ manual response choice reaction time.

\section{Analysis of Visual Search Data}

Visual search patterns were analyzed in a semiautomatic method involving a video player, a computer, and a video digitizing board. Each trial was digitized frame by frame in order to obtain $x$ and $y$ coordinates for the subject's visual fixation point. After previous research, a fixation was operationally defined as a condition in which the eye remained stationary for a period equal to, or in excess of, six video frames or $120 \mathrm{msec}$ (see Williams et al., 1994).

Eye movements were then analyzed to obtain the following information:

Fixation order. The fixation order was defined as the search sequence or pattern used by the subjects. Previous research indicated that the most important search pattern when attempting to anticipate pass destination was to alternate fixations between the box and other areas of the display (see Williams et al., 1994). This search strategy was regarded as being advantageous because it enhanced the subjects' awareness of players' positions and movements, thus increasing their appreciation of possible passing options (Williams et al., 1994). The mean number of times that this search pattern was used per trial was employed to determine differences in search order across the two conditions.

Fixation location. The fixation location referred to the area of the display on which the eye fixated when predicting pass destination. As stated previously, for a meaningful analysis of fixation location, the screen was divided into three areas: box, right, and left. This analysis provided percentage values for the mean time spent per trial within each of the three locations.

Search rate. The search rate was represented by the mean number of visual fixations per trial.

\section{Analysis of Verbal Report Data}

The verbal report data were analyzed using a video player to obtain data on the search order, location of visual attention, and search rate.

Search order. The search order was defined as the mean number of verbalized shifts in attention per trial from the box to the right or left side of the screen then back to the box.

Location of visual attention. The location of visual attention was regarded as the number of verbalizations per trial to each of the three separate locations (i.e., box, right, and left side of the screen) as a percentage of the total number of verbalizations.

Search rate. This was represented by the mean number of verbalizations made by the subject per trial.

\section{Results}

\section{Anticipation Test}

Performance on the anticipation test was analyzed using a two-way split-plot multivariate analysis of covariance (MANCOVA). In this analysis, experience (experienced/less experienced) was the between-subjects factor, condition (verbal report/eye movement) was the within-subjects variable, VRE and RT were the depen- dent variables, and verbal and manual CRT were the covariates. ${ }^{3}$ Mean group performance scores across the two conditions are shown in Table 1 . The results showed no significant differences in performance on the anticipation test across the two conditions [Wilks's lambda $=0.92$, $F(2,16)=0.68, p>.05]$. Separate univariate analyses of the two dependent variables revealed no significant differences in either VRE measures $[F(1,17)=1.39, p>$ $.05]$ or RT measures $[F(1,17)=0.76, p>.05]$. However, the MANCOVA did reveal a significant difference in performance between the two groups [Wilks's lambda $=0.36$, $F(2,16)=13.73, p<.001]$. Univariate analyses showed that the experienced soccer players had quicker RT values $[F(1,17)=15.01, p<.01]$. There were no differences in VRE between groups $[F(1,17)=0.04, p>.05]$. Finally, the MANCOVA showed that there was no significant group $\times$ condition interaction [Wilks's lambda $=$ $0.87, F(2,16)=1.23, p>.05]$.

\section{Comparison Between Eye Movements and Verbal Reports}

Search order. Differences in the frequency of alternation of visual fixations and/or visual attention between the box and the right or left side of the screen were examined using a split-plot analysis of variance (ANOVA) in which group (experienced/less experienced) was the between-subjects variable and condition (eye movement/ verbal report) was the within-subjects factor. The results are represented in Table 2 . Findings showed no significant differences between groups $[F(1,18)=2.62, p>$ $.05]$. However, there was significant main effects for condition $[F(1,18)=38.16, p<.01]$ and for the group $\times$ condition interaction $[F(1,18)=4.60, p<.05]$. Post hoc Newman-Keuls analyses on the group $\times$ condition interaction showed that this search pattern was more evident for the eye-movement data than for the verbal re-

Table 2

Differences Between the Eye-Movement and Verbal Report Conditions in the Number of Times Per Trial That Subjects Alternated Their Search Strategy Between the Box and the Right and the Left Sides of the Screen in the 11-Versus-11 Situations

\begin{tabular}{lccccc}
\hline & \multicolumn{2}{c}{$\begin{array}{c}\text { Eye-Movement } \\
\text { Condition }\end{array}$} & & \multicolumn{2}{c}{$\begin{array}{c}\text { Verbal Report } \\
\text { Condition }\end{array}$} \\
\cline { 2 - 3 } \cline { 5 - 6 } \multicolumn{1}{c}{ Group } & $M$ & $S D$ & & $M$ & $S D$ \\
\hline Experienced $(n=10)$ & 2.55 & 0.47 & & 1.61 & 0.34 \\
Less experienced $(n=10)$ & 2.11 & 0.31 & & 1.65 & 0.38 \\
\hline
\end{tabular}


port data $(p<.05)$. Furthermore, the eye-movement data showed that experienced soccer players scanned more frequently from the box, to the right or left side of the screen, then back to the box $(p<.01)$. In contrast, the verbal report data revealed no differences between groups in the frequency of this search pattern $(p>.05)$.

Fixation location versus visual attention. Differences in visual orientation (as implied from the eye-movement data) and visual attention (as inferred from verbal reports) were examined using a split-plot ANOVA in which group (experienced/less experienced) was the betweensubjects variable and condition (verbal report/eye movement) and the three locations (box/right/left) were the within-subjects factors. The findings are represented in Figure 2. The results showed no significant differences in the group $[F(1,18)=2.19, p>.05]$ or condition $[F(1,18)$ $=0.00, p>.05]$ main effects. However, a significant difference was found in the location main effect $[F(2,36)=$ 97.64, $p<.0001]$. Also, significant interactions were shown between group and location $[F(2,36)=16.23, p<$ $.0001]$ and between condition and location $[F(2,36)=$ $9.07, p<.0001]$. Post hoc Newman-Keuls analysis on the group $\times$ location interaction revealed a significant difference between the experienced and less experienced groups in the distribution of visual fixations and verbalizations to the box area $(p<.001)$ and to the left side of the screen $(p<.05)$. The less experienced group spent significantly more time per trial fixating or visually attending to (i.e., verbalizing) information inside the box area and less time on the left side of the screen. NewmanKeuls analysis on the condition $\times$ location interaction revealed that more time was spent fixating inside the box area during the eye-movement condition, compared with the amount of time the subjects spent visually attending to this area according to the verbal report approach $(p<$ $.01)$. No significant differences were evident between eye

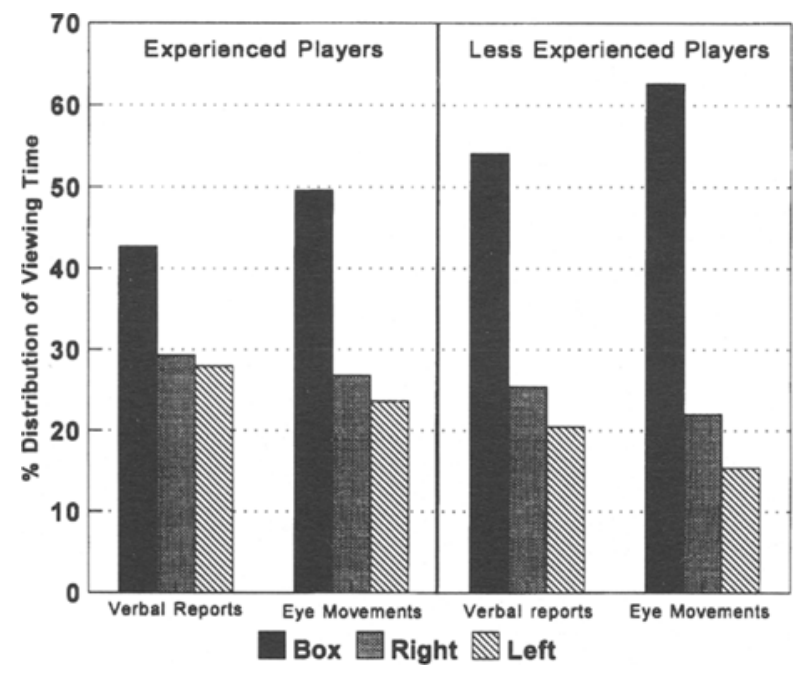

Figure 2. Distribution of verbal reports and visual fixations to selected areas of the display in terms of percentage of total viewing time in 11-versus-11 situations. movements and verbal reports in assessing when the subjects were visually fixating or attending to information from the right side of the screen $(p>.05)$. The mean value for the amount of time fixating inside the box for the eye-movement condition was $56.56 \%(S D=10.27)$; the mean value for the amount of time attending to information inside the box area for the verbal report condition was $47.69 \%(S D=9.62)$.

The relationships between eye movements and verbal reports in identifying differences in visual attention were examined by Pearson product-moment correlations. Moderately high and significant correlations were obtained between the two methods with regard to the amount of time in the box $(r=.62, p<.01)$ and the left side of the screen $(r=.72, p<.01)$ respectively. A moderately low and nonsignificant correlation was found for the right side of the screen $(r=.29, p>.05)$.

Search rate. Differences in search rate between the two conditions were examined using a split-plot ANOVA where group (experienced/less experienced) was the between-subjects factor, and condition (eye movement/ verbal report) was the within-subjects variable. Findings showed no significant differences in the effects of group $[F(1,18)=3.85, p>.05]$ and condition $[F(1,18)=1.77$, $p>.05]$, and no significant interaction $[F(1,18)=2.97$, $p>.05]$. The experienced players employed a comparable number of visual fixations prior to response in the eye-movement condition $(M=10.30, S D=0.97)$ and in the verbal report condition $(M=10.21, S D=1.63)$. Similarly, there were no differences in the number of visual fixations $(M=8.72, S D=0.94)$ and the number of verbalizations $(M=10.12, S D=1.35)$ employed by the less experienced group.

\section{Discussion}

The results showed that, regardless of experience level, there were no significant differences in performance on the 11-versus-11 anticipation test across the two conditions. Thus, requiring the subjects to concurrently verbalize the area of the display that they were extracting information from had no effect on task performance. The finding that concurrent verbalization does not result in reactivity contradicted previous research. For example, several studies have demonstrated that concurrent verbalization seems to exert a general "slowingdown" effect on task-directed processes (e.g., Brinkman, 1993; McGeorge \& Burton, 1989; Toms, 1992). However, previous research has tended to use more static tasks requiring subjects to describe the cognitions and mental processes used during task performance (e.g., see Brinkman, 1993). The argument is that asking subjects to report their cognitive processes places a greater demand on the limited-capacity short-term memory, thus slowing down task-directed processes (Ericsson \& Simon, 1993). This suggests that the type of task influences the potential for reactivity due to concurrent verbalization. In the present experiment, the subjects were asked to verbalize the perceptual strategy they were using during a dynamic sport situation. 
The results demonstrated many similarities between verbal reports and eye movements as methods of assessing visual attention during performance in a team game context. For example, there were no differences between the two methods in identifying the location of visual attention and/or fixation. The eye-movement data indicated that the less experienced group spent more time fixating inside the box area than did the experienced group, whereas the verbal report data provided supporting evidence to suggest that the less experienced players expended more time extracting information from this area of the display. That is, the less experienced players appeared to be guilty of "ball watching." Moreover, both methods showed that the experienced group spent more time visually fixating on, and attending to, other areas of the display, such as the positions and movements of players (see Williams et al., 1994). This suggests that the experienced players considered areas other than the box (i.e., ball/ball passer) to be more informative. ${ }^{4}$ The correlation coefficients of $.72, .29$, and .62 between fixations on the box, right, and left side of the screen, respectively, provide further support for the similarity of the data. Therefore, in this experiment, eye-movement recording provided a valid measure of attentional allocation and information extraction. Since verbal reports are assumed to provide a measure of overt cognitive processing, the explicit relationship between these two methods verifies the use of eye-fixation data as a measure of selective attention in this task.

However, an interesting difference between the two methods was that both groups spent more time fixating inside the box area in the eye-movement condition. An explanation may be that because the subjects were continually verbalizing "box" during the verbal report condition, they may have become more aware of the restricted nature of their search pattern. Consequently, they may have been encouraged to try and extract information from other display areas. The suggestion is that the verbalization procedure affected the automation of the search process and influenced the choice of performance strategy. Another possibility is that the subjects were using peripheral vision to extract information from the display during the eye-movement condition. This would have inflated the importance of information from the box area during task performance in this condition.

Finally, differences were evident in search order between the two conditions. The eye-movement condition revealed a significant difference between the two groups with regard to the frequency of alternation of fixations between the box and other areas of the display. The experienced group used this strategy an average of 2.55 $(S D=0.47)$ times per trial, and the less experienced group used this strategy an average of $2.11(S D=0.31)$ times per trial. In contrast, the verbal report condition indicated much lower frequencies of alternation of attention for the experienced group $(M=1.61, S D=0.34)$ and the less experienced group $(M=1.65, S D=0.38)$. This suggested that the eye-movement technique was a more sensitive measure of search order. The subjects may have had some difficulty in verbally indicating every shift in attentional allocation. This may particularly be the case in dynamic situations when brief and rapid alternation of attention is required (i.e., attention switching).

\section{EXPERIMENT 2}

Experiment 2 examined the relationship between concurrent verbal reports and eye-movement recording as measures of selective attention in 3-versus-3 soccer simulations. Previous research has shown that experienced soccer players rely on peripheral vision to extract taskspecific information in these situations (see Williams et al., 1995a, 1995b). When a film occlusion technique was employed to mask information pickup from areas other than the ball/ball passer, there was a greater decrement in the experienced players' performance compared with that of their less experienced counterparts. Since there were no differences between groups in the orientation of foveal vision to these display areas, this indicated that the experienced players extracted more information through nonfoveal stimulation. Therefore, these 3-versus3 situations differed from those employed in Experiment 1 because they required subjects to make greater use of peripheral vision in selective information pickup. Consequently, it was hypothesized that there would be a much weaker relationship between foveal orientation (as implied from eye-fixation data) and concurrent verbal reports.

\section{Method}

\section{Subjects}

The subjects were 12 experienced male soccer players $(M$ age $=$ 24.0 years, $S D=4.1)$ and 12 less experienced male soccer players ( $M$ age $=23.3$ years, $S D=4.0$ ). The experienced players had been playing soccer for 13.4 years, including an average of 754 ( $S D=$ 179.5) competitive matches. They included 5 professional soccer players and 7 semiprofessional players. The less experienced group had been playing soccer for an average of 4.1 years $(S D=$ $2.5)$ and had experienced an average of 65 matches $(S D=52.7)$. They were all recreational soccer players. The subjects gave their informed consent to participate in the experiment.

\section{Test Film}

The subjects were presented with 20 offensive soccer sequences. These were 3 -versus- 3 situations, where the subjects were required to imagine themselves as a covering defender or sweeper. Each sequence included two central defenders marking two offensive players and a defensive midfield player marking an offensive midfield player. The film clips lasted approximately $6 \mathrm{sec}$ and included an offensive pattern of play, ending with a pass being made to the subject's right or left side, the ball being played over the subject's head into the space behind him, or an offensive player dribbling the ball directly toward the subject. Care was taken to ensure that the final pass or dribble was distinct, thus necessitating a definite response from the subject viewing the film sequence. With this in mind, the offensive midfield player who made the eventual pass or dribble was surrounded by a black reference square during the entire trial so that he could be clearly highlighted. The reference square subtended a visual angle of approximately $8^{\circ}$ in the horizontal direction and $7^{\circ}$ in the vertical direction. The square did not mask the player's actions on screen. An intertrial interval of $8 \mathrm{sec}$ was built into the film construction. 
The offensive patterns were selected from actual soccer matches and then realistically simulated by the offensive and defensive players. The simulation was filmed from a position behind the players $(5 \mathrm{~m})$ using a video camera, an $8-\mathrm{mm}$ focal length lens, and a video recorder. This position provided a visual display most representative of a central defensive player's view of the game. Three experienced soccer coaches viewed the offensive sequences to ensure that each film clip was realistic and representative of actual game play.

\section{Apparatus}

The apparatus was the same as that used in Experiment 1.

\section{Procedure}

Eye-movement condition. Prior to presentation of the test film, the subjects' CRT was measured. An empty soccer field was presented as the display. Following a random foreperiod, the subjects were required to respond as quickly and as accurately as possible when a stimulus was presented. This involved the presentation of a red circle in the right, left, top, or bottom of the screen. The subject's task was to step onto a response pad corresponding to the position of the red circle (i.e., right, left, front, or back response pad). The response pads were positioned on the floor a distance of $1 \mathrm{~m}$ away from the subject. The subjects were presented with 3 practice trials and 18 test trials. CRT was measured as the time from the presentation of the stimulus to the completion of the subject's response. ${ }^{5}$ This task served to familiarize the subjects with the response required in the anticipation test and provided the baseline measure of CRT used as a covariate in the subsequent performance analysis.

Following the CRT test, the eye-movement system was fitted on the subject's head. The habituation and calibration procedure for the eye-movement system was the same as that in Experiment 1.

The test film was then presented using a video projection system and a $3.5 \times 3 \mathrm{~m}$ screen. The subjects stood $5 \mathrm{~m}$ away from the screen so that the film image subtended a visual angle of approximately $40^{\circ}$ in the horizontal direction and $35^{\circ}$ in the vertical direction. The subjects viewed each pattern of play as it developed on screen and were required to respond as quickly and accurately as possible by moving right, left, forward, or backward in response to each action sequence. The subjects were required to step on the same response pads used in the CRT test. Immediately following their response, the film was occluded in order to prevent the subjects from gaining feedback on task performance. The time from the start of the film clip to the completion of the response was recorded using pressure-sensitive pads and a microcomputer. After the first 10 film trials, the subjects were given a 2 -min rest period during which the calibration of the eye-movement system was checked. The entire test session lasted approximately $30 \mathrm{~min}$.

Verbal report condition. The subjects viewed the same test film used in the eye-movement condition. However, the sequence of presentation of film clips was altered to minimize order effects. As in Experiment 1 , conditions were counterbalanced to reduce learning biases. The same test procedure used in the eye-movement condition was adopted for the verbal report condition. While each action sequence unfolded, the subjects were required to continuously verbalize the area of the display from which they were extracting information. As in Experiment 1, the subjects were required to verbally indicate when they were allocating attention to the box, left, or right side of the screen. The practice and habituation process was the same as that in Experiment 1. Also, the subjects' verbalizations were recorded and analyzed using the same procedure adopted in Experiment 1. The entire test session lasted for $30 \mathrm{~min}$ on average.

\section{Dependent Variables and Analysis for the 3-Versus-3 Anticipation Test}

The reactivity of the verbal report technique was tested by comparing performance on the anticipatıon test in the verbal report and eye-movement conditions. The following dependent measures were obtained:

Total response time (TRT). The TRT was the time (in milliseconds) from the start of the film clip to the completion of the subject's response.

Verbal response error (VRE). The VRE was obtained by comparing the subjects' verbal response to the actual pass destination (in percentages)

\section{Analysis of Visual Search Data}

Visual search patterns were analyzed manually using the frameby-frame facility on a video player. Eye movements were analyzed to obtain the same information as in Experiment 1.

\section{Analysis of Verbal Report Data}

The verbal report data were analyzed to obtain the same information used in Experiment 1.

\section{Results}

\section{Anticipation Test}

Performance on the anticipation test was analyzed using a split-plot two-way MANCOVA. In this analysis, experience (experienced/less experienced) was the betweensubjects factor, condition (verbal report/eye movement) was the within-subjects variable, VRE and TRT were the dependent variables, and CRT was the covariate. ${ }^{6}$ Mean group performance scores across the two conditions are shown in Table 3 . The results showed a significant difference in performance on the anticipation test across the two conditions [Wilks's lambda $=0.42, F(2,20)=13.57$, $p<.0001]$. Separate univariate analyses of the two dependent variables revealed a significant difference in the TRT variable $[F(1,22)=20.91, p<.001]$. There were no differences on the VRE variable $[F(1,22)=1.55, p>.05]$ Also, the MANCOVA showed a significant difference in performance between the two groups [Wilks's lambda = $0.57, F(2,20)=7.28, p<.01]$. Univariate analyses showed that the experienced soccer players had quicker TRT values $[F(1,21)=10.24, p<.01]$. There were no differences in VRE between groups $[F(1,21)=1.17, p>.05]$. Fi-

Table 3

Differences in Anticipatory Performance in 3-Versus-3 Situations Between the Experienced and Less Experienced Soccer Players Across the Eye-Movement and Verbal Report Conditions

\begin{tabular}{|c|c|c|c|c|c|c|c|c|c|c|c|c|}
\hline \multirow[b]{3}{*}{ Group } & \multicolumn{6}{|c|}{ Eye-Movement Condition } & \multicolumn{6}{|c|}{ Verbal Report Condition } \\
\hline & \multicolumn{2}{|c|}{ CRT (msec) } & \multicolumn{2}{|c|}{ VRE $(\%)$} & \multicolumn{2}{|c|}{ TRT (msec) } & \multicolumn{2}{|c|}{ CRT (msec) } & \multicolumn{2}{|c|}{ VRE (\%) } & \multicolumn{2}{|c|}{ TRT (msec) } \\
\hline & $M$ & $S D$ & $M$ & $S D$ & $M$ & $S D$ & $M$ & $S D$ & $M$ & $S D$ & $M$ & $S D$ \\
\hline & 52 & 49 & 21 & 11 & $426 ?$ & 22 & 52 & 4 & 22 & 13.7 & 4, & 32 \\
\hline Less experienced $(n=12)$ & 518 & 54 & 20.1 & 14.9 & 4,484 & 225 & 518 & 54 & 15.8 & 9.3 & 4,792 & 229 \\
\hline
\end{tabular}

Note - CRT $=$ choice reaction time; VRE $=$ verbal response error; TRT $=$ total response time. 
nally, the MANCOVA showed that there was no significant group $\times$ condition interaction [Wilks's lambda $=$ $0.89, F(2,20)=1.21, p>.05]$.

\section{Comparison Between Eye Movements and Verbal Reports}

Search order. Differences in the frequency of alternation of visual fixations and/or visual attention between the box and the right or left side of the screen were examined using a split-plot ANOVA. In this analysis, group (experienced/less experienced) was the between-subjects variable, and condition (eye movement/verbal report) was the within-subjects variable. Findings showed no significant differences between groups $[F(1,22)=0.23, p>$ $.05]$ or across conditions $[F(1,22)=0.29, p>.05]$. Also, there was no group $\times$ condition interaction $[F(1,22)=$ $0.74, p>.05]$. The experienced players used this search pattern on average $1.03(S D=0.39)$ times per trial in the eye-movement condition and $1.06(S D=0.64)$ times per trial in the verbal report condition. Similarly, the less experienced players did not use this search pattern more frequently in the eye-movement condition $(M=1.06$, $S D=0.22$ ) than they did in the verbal report condition $(M=0.9, S D=0.42)$.

Fixation location versus visual attention. Differences in visual orientation (as implied from eye-movement data) and visual attention (as inferred from verbal reports) were examined using a split-plot ANOVA. In this analysis, group (experienced/less experienced) was the between-subjects variable and condition (verbal report/eye movement) and the three locations (box/right/left) were the within-subjects variables. The findings are represented in Figure 3. The results showed no significant difference in the group main effect $[F(1,22)=0.10, p>.05]$. However, significant differences were shown for the main ef-

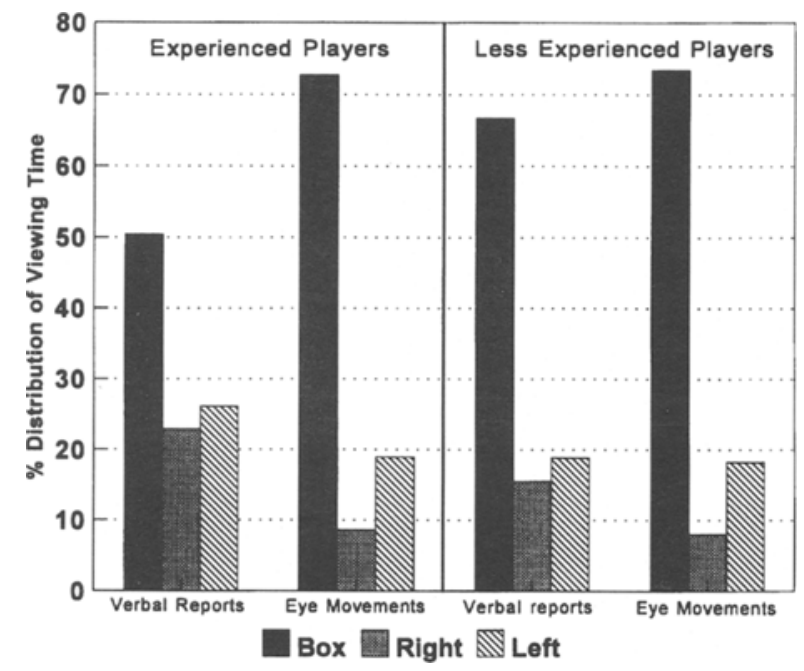

Figure 3. Distribution of verbal reports and visual fixations to selected areas of the display in terms of percentage of total viewing time in 3-versus-3 situations. fects of condition $[F(1,22)=26.76, p<.0001]$ and location $[F(2,44)=74.07, p<.00001]$. Also, significant interactions were established for group $\times$ location $[F(2,44)=$ $74.07, p<.00001]$ condition $\times$ location $[F(2,44)=80.85$, $p<.00001]$ and group $\times$ condition $\times$ location $[F(2,44)=$ $80.85, p<.01]$. Post hoc Newman-Keuls analysis on the group $\times$ condition $\times$ location interaction showed that there were no significant differences in the distribution of eye fixations compared with that of verbal reports for the less experienced subjects $(p>.05)$. That is, there were no differences between eye fixations and verbal reports in their ability to identify important display areas for this subject group. However, both methods indicated that the less experienced players spent more time visually fixating and/or verbalizing information in the box area than they did in the right and left sides of the display $(p<.001)$. In contrast, differences were found between eye fixations and verbal reports for the experienced players $(p<.001)$. In the verbal report condition, the experienced players spent less time per trial visually attending to (i.e., verbalizing) information inside the box area and more time on the right side of the screen $(p<.001)$. In the eye-movement condition, there were no differences in fixation location or visual attention between groups; in the verbal report condition, the less experienced players spent more time fixating inside the box area and less time on the right side of the screen $(p<.001)$. The mean value for the amount of time visually fixating inside the box in the eye-movement condition was similar for the experienced players $(M=72.45 \%, S D=6.51)$ and the less experienced players $(M=73.78 \%, S D=6.58)$. In contrast, the mean time visually attending inside the box in the verbal report condition was different for the experienced players $(M=50.92 \%, S D=11.02)$ and the less experienced players $(M=66.74 \%, S D=11.42)$.

The relationships between the eye movements and verbal reports in identifying differences in visual attention were examined by Pearson product-moment correlations. Nonsignificant correlations were obtained between the two methods with regard to the amount time spent in the box $(r=.01, p>.01)$ and in the left side $(r=$ $.35, p>.05)$ and the right side $(r=.37, p>.05)$ of the screen.

Search rate. Differences in search rate between the two conditions were examined using a split-plot ANOVA where group (experienced/less experienced) was the between-subjects factor and condition (eye movement/ verbal report) was the within-subjects variable. Findings showed no significant group main effect $[F(1,22)=3.04$, $p>.05]$. However, significant differences were obtained for the main effect of condition $[F(1,22)=63.89, p<.0001]$ and the group $\times$ condition interaction $[F(1,22)=4.42$, $p<.05]$. The results are highlighted in Table 4. Post hoc Newman-Keuls analysis on the group $\times$ condition interaction indicated that both groups of subjects made more verbalizations than visual fixations $(p<.001)$. Furthermore, although there were no differences in the number 
Table 4

Differences Between Groups in the Number of Visual Fixations Per Trial Compared With the Number of Verbalizations Per Trial in the 3-Versus-3 Situations

\begin{tabular}{lccccc} 
& \multicolumn{2}{c}{$\begin{array}{c}\text { Eye-Movement } \\
\text { Condition }\end{array}$} & & \multicolumn{2}{c}{$\begin{array}{c}\text { Verbal Report } \\
\text { Condition }\end{array}$} \\
\cline { 2 - 3 } \multicolumn{1}{c}{ Group } & $M$ & $S D$ & & $M$ & $S D$ \\
\hline Experienced $(n=12)$ & 4.17 & 0.62 & & 6.16 & 1.35 \\
Less experienced $(n=12)$ & 4.02 & 0.90 & & 7.44 & 1.48 \\
\hline
\end{tabular}

of visual fixations across groups, the less experienced players made more verbalizations per trial than did the experienced subjects $(p<.05)$.

Although the less experienced players had more verbalizations per trial, it was thought that they would alter their locus of attention more frequently. The frequency of alternation of attention was determined by dividing the number of changes in the location of attention per trial by the total number of verbalizations and then multiplying by 100 to obtain a percentage value. This variable was then analyzed using an independent-samples $t$ test. The results showed that there was a significant difference between groups $[t(24)=2.10, p<.05]$, with more of the experienced players' verbalizations $(M=$ $71.45 \%, S D=12.64$ ) being directed toward different display areas compared with those of the less experienced subjects $(M=58.44 \%, S D=14.64)$.

\section{Discussion}

There were significant differences in performance on the 3-versus-3 anticipation test across the two conditions. That is, requiring the subjects to concurrently verbalize the area of the display from which they were extracting information had a detrimental effect on performance. This supported previous research and suggested that concurrent verbalization exerted a general slowing-down effect on task-directed processes (e.g., Brinkman, 1993; McGeorge \& Burton, 1989; Toms, 1992). However, the results contradicted those obtained in Experiment 1.? Since previous research has demonstrated that subjects use peripheral vision to extract information in these 3versus-3 simulations (see Williams et al., 1995a, 1995b), perhaps requiring subjects to verbalize this information interferes with task performance. The extraction of information from the peripheral visual field is often subconscious; therefore, asking subjects to consciously verbalize this information may disrupt the automaticity of the task, resulting in reactivity. In contrast, the subjects used foveal vision to extract task-specific information in Experiment 1 (see Williams et al., 1994). Since extracting information through foveal vision is normally a conscious, attention-demanding process, requiring subjects to verbally report this information does not result in reactivity. This argument is supported by the fact that decrements in performance are observed when subjects are required to use implicit or tacit knowledge in a conscious or explicit manner (for a more detailed review, see Myers \& Davids, 1993).
The results also showed that there were differences between verbal reports and eye movements in their ability to identify the locus of visual attention in these 3-versus3 situations. The eye-movement data indicated that there were no differences in the distribution of visual fixations to selected areas of the display. According to this method, the subjects fixated primarily on the box, with infrequent fixations to the right or left of the screen. However, the verbal report condition showed that the experienced players distributed their attention evenly between the box and the right and left side of the screen. In the verbal report condition, the experienced players were attending to information inside the box area for a shorter time period $(M=50.92 \%)$ than they were in the eyemovement condition $(M=72.45 \%)$. The suggestion is that, although the experienced players were visually fixating inside the box area in the eye-movement condition, they were using peripheral vision to extract information from other areas of the display. They seemed able to use the box as a visual pivot or central reference point while simultaneously scanning peripheral vision for the positions and movements of players. This more extensive search strategy was highlighted by the fact that, although they had fewer verbalizations per trial, $71.44 \%$ of these comments involved a change in the locus of attention, compared with $58.44 \%$ for the less experienced players. In contrast, there were no differences between eye movements and verbal reports for the less experienced players. They made less use of peripheral vision, preferring instead to use foveal vision to extract information from the ball or the player in possession of the ball. These findings supported earlier research that used a film occlusion technique to mask information pickup from areas other than the ball/ball passer (see Williams et al., 1995b). In sum, in this experiment, eye-movement recording provided a poor measure of attentional allocation and information extraction. ${ }^{8}$

\section{CONCLUSIONS AND SUMMARY}

These experiments showed that the validity of eyemovement recording as a method of determining differences in selective attention is dependent on subject experience and the type of task undertaken. It appears that visual orientation (as implied from eye fixations) is indicative of information pickup and cue usage when the task necessitates that subjects use foveal vision to extract information from the display. For example, in the 11versus- 11 simulations, proficiency-related differences in visual search strategy were observed using both concurrent verbal report and eye-movement methods. In these simulations, the subjects had to be aware of the positions and movements of a large number of players, and, consequently, foveal vision was primarily used to pickup information from the display (see Williams et al., 1994). Furthermore, neither procedure induced reactivity, suggesting that either method can be employed to assess cue usage in such situations. However, since eye-movement recording provided a more sensitive measure of search 
order, it may offer some advantages over verbal protocols when subjects are required to rapidly alternate the location of attention in dynamic task simulations (i.e., attention switching). In contrast, Experiment 2 suggested that eye fixations are a poor indicator of visual search strategy and selective attention when the task requires subjects to use peripheral vision to extract information from the display. The results showed that, although concurrent verbal reports resulted in reactivity, they provided a more valid measure of selective attention in the 3 -versus-3 simulations. The efficacy of eye-movement recording appears to be dependent on two mediating factors: (1) the role of peripheral vision in extracting taskspecific information, and (2) the experience level of the subjects.

In Experiment 2, the experienced soccer players visually fixated on the box area because it was the most appropriate reference point for picking up and organizing information from the periphery (see Ripoll, 1991; Williams et al., 1995a, 1995b). This type of approach has several advantages. First, because saccades are inactive periods of information processing (Abernethy, 1985), it enables subjects to use a more efficient search pattern involving fewer fixations of longer duration. Second, it allows subjects to switch their attention more rapidly from one information source to another, which is regarded as being important in dynamic contexts such as driving a car or landing helicopters (Berbaum et al., 1991; Hughes \& Cole, 1986). The experienced performers' ability to effectively use peripheral vision in these dynamic contexts highlights the advantages of using more direct measures of information extraction to assess search strategy. Also, concurrent verbal reports are inexpensive and data can be analyzed rapidly, thus enabling large subject samples to be tested. However, further work is required to identify the specific utility of these procedures in the human performance area. In particular, research must examine whether concurrent verbalization protocols can solve more sophisticated visual search problems. This research required subjects only to verbalize fixations to three areas of the screen (i.e., box, right, and left side). Future research should attempt to enhance the sensitivity of such protocols, thus enabling a more extensive profile of expert-novice differences in search strategy. However, care must be taken when using more refined procedures, because increasing the number of possible areas that can be verbalized may affect the automaticity of the search process, thus increasing task reactivity. Perhaps verbal report protocols may be limited to identifying more general, rather than specific, information sources.

In conclusion, this research examined the validity of eye fixations and verbal reports as methods of assessing differences in selective information pickup during dynamic sport simulations. The results showed that both eye fixations and verbal reports provide valid measures of selective attention in tasks that require information extraction via foveal or central vision. This category of tasks may include computer programming, machine operation, industrial inspection, and radiography. In con- trast, when the task requires the subjects to extract taskspecific information through peripheral vision, verbal report protocols may provide a more veridical measure of information extraction. This category may include dynamic tasks such as driving a car, flying, landing helicopters, and sport simulations. The experienced performers' ability to extract information through nonfoveal stimulation in these contexts highlights the limitations of relying solely on eye-movement recording to examine differences in visual search strategy and selective information pickup.

\section{REFERENCES}

ABERNETHY, B. (1985). Cue usage in "open" motor skills: A review of available procedures. In D. G. Russell \& B. Abernethy (Eds.), Motor memory and control: The Otago Symposium (pp. 110-122). Dundein, NZ: Human Performance Associates.

ABERNETHY, B. (1988). Visual search in sport and ergonomics: Its relationship to selective attention and performer expertise. Human Performance, 4, 205-235.

ABERNETHY, B. (1990). Expertise, visual search, and information pickup in squash. Perception, 19, 63-77.

APPLIED SCIENCE LABORATORIES (1992). Eye tracking systems handbook. Bedford, MA: Author.

BAINBRIDGE, L. (1990). Verbal protocol analysis. In J. R. Wilson \& E. N. Corlett (Eds.), Evaluation of human work: A practical ergonomic methodology (pp. 161-179). London: Taylor \& Francis.

Berbaum, K. S., Kennedy, R. S., \& Hettinger, L. J. (1991). Visual tasks in helicopter shipboard landing. Applied Ergonomics, 22, 231239.

BRINKMAN, J. A. (1993). Verbal protocol accuracy in fault diagnosis. Ergonomics, 36, 1381-1397.

Buckolz, E., Prapavesis, H., \& Fairs, J. (1988). Advance cues and their use in predicting tennis passing shots. Canadian Journal of Sport Sciences, 13, 20-30.

DAvids, K. (1984). The role of peripheral vision in ball games: Some theoretical and practical notions. Physical Education Review, 7, 26-40.

ERICSSON, K. A., \& Oliver, W. L. (1989). A methodology for assessing the detailed structure of memory skills. In A. M. Colley \& J. R. Beech (Eds.), Acquisition and performance of cognitive skills (pp. 193-216). New York: Wiley.

ERICsson, K. A., \& SimON, H. A. (1980). Verbal reports as data. Psychological Review, 87, 215-251.

ERICSSON, K. A., \& Simon, H. A. (1993). Protocol analysis: Verbal reports as data. Cambridge, MA: MIT Press.

Green, A. J. (1995). Verbal protocol analysis. The Psychologist, 8, 126-129.

HELLA, F. (1984). Is the analysis of eye movement recording a sufficient criterion for evaluating automobile instrument panel design? In J. K. O'Regan \& A. Levy-Schoen (Eds.), Eye movements: From physiology to cognition (pp. 551-561). Amsterdam: North-Holland.

HuGHES, P. K., \& COLE, B. L. (1986). What attracts attention when driving? Ergonomics, 29, 377-391.

JoNIDES, J. (1981). Voluntary versus automatic control over the mind's eye. In J. Long \& A. Baddeley (Eds.), Attention and performance IX (pp. 187-203). Hillsdale, NJ: Erlbaum.

JORGENSEN, A. H. (1990). Thinking-aloud in user interface design: A method promoting cognitive ergonomics. Ergonomics, 33, 501-507.

KoubeK, R. J., \& SAlvendy, G. (1991). Cognitive performance of super-experts on computer program modification tasks. Ergonomics, 34, 1095-1112.

Kundel, H. L., Nodine, C. F., \& Toto, L. (1984). Eye movements and the detection of lung tumours in chest images. In A. G. Gale \& F. Johnson (Eds.), Theoretical and applied aspects of eye movement research (pp. 297-304). Amsterdam: North-Holland.

Le Plat, H., \& Hoc, J. M. (1981). Subsequent verbalization in the study of cognitive processes. Ergonomics, 24, 743-756.

McGeorge, P., \& Burton, A. M. (1989). The effects of concurrent 
verbalization on performance in a dynamic systems task. British Journal of Psychology, 80, 455-465.

Myers, C., \& Davids, K. (1993). Tacit skill and performance at work. Applied Psychology: An International Perspective, 42, 117-137.

Neumann, O., van der Heijden, A. H. C., \& Allport, D. A. (1986). Visual selective attention: Introductory remarks. Psychological Research, 48, 185-188.

NisBert, R. E., \& WILSON, T. D. (1977). Telling more than we can know: Verbal reports on mental processes. Psychological Review, 84, 231-259.

Paillard, J. (1982). The contribution of peripheral and central vision to visually guided reaching. In D. J. Ingle, M. A. Goodale, \& R. J. Mansfield (Eds.), Analysis of visual behavior (pp. 367-385). Cambridge, MA: MIT Press.

PAPIN, J. M. (1984). Use of the NAC eye mark recorder to study visual strategies of military aircraft pilots. In A. G. Gale \& F. Johnson (Eds.), Theoretical and applied aspects of eye movement research (pp. 367-371). Amsterdam: North-Holland.

Papin, J. M., Metges, P., \& AmalberTi, R. (1984). Use of NAC eye mark by radiologists. In A. G. Gale \& F. Johnson (Eds.), Theoretical and applied aspects of eye movement research (pp. 323-330). Amsterdam: North-Holland.

REED, N. E., \& Johnson, P. E. (1993). Analysis of expert reasoning in hardware diagnosis. International Journal of Man-Machine Studies, 38, 251-280.

Remington, R. W. (1980). Attention and saccadic eye movements. Journal of Experimental Psychology: Human Perception \& Performance, 6, 726-744.

RIPOLL, H. (1991). The understanding-acting process in sport: The relationship between the semantic and the sensorimotor visual function. International Journal of Sport Psychology, 22, 221-243.

RoCKWELL, T. (1972). Skills, judgement and information acquisition in driving. In T. W. Forbes (Ed.), Human factors in highway traffic safety research (pp. 133-164). New York: Wiley.

RusSo, J. E., JohnSON, E. J., \& STEPHENS, D. L. (1989). The validity of verbal protocols. Memory \& Cognition, 17, 759-769.

Sanders, A. F., \& Houtmans, M. J. M. (1985). Perceptual processing modes in the functional visual field. Acta Psychologica, 58, 251-261.

Shulman, G. L., Remington, R. W., \& MCLEan, J. P. (1979). Moving attention throughout visual space. Journal of Experimental Psychology: Human Perception \& Performance, 5, 522-526.

STAGER, P., \& ANGUS, R. (1978). Locating crash sites in a simulated air-to-ground visual search. Human Factors, 20, 453-466.

Toms, M. (1992). Verbal protocols: How useful are they to cognitive ergonomists? In E. J. Lovesey (Ed.), Contemporary ergonomics (Proceedings of the Ergonomics Society's 1992 Annual Conference, pp. 316-321). London: Taylor \& Francis.

Treisilian, J. R. (1995). Perceptual and cognitive processes in time-tocontact estimation: Analysis of prediction-motion and relative judgment tasks. Perception \& Psychophysics, 57, 231-245.

Tyldesley, D. A., Bootsma, R. J., \& Bomhoff, G. T. (1982). Skill level and eye movement patterns in a sport orientated reaction time task. In H. Rieder, K. Bos, H. Mechling, \& K. Reischle (Eds.) Motor learning and movement behaviour: Contribution to learning in sports (pp. 290-296). Cologne: Hoffmann.

van der Hejuden, A. H. C. (1986). On selection in vision. Psychological Research, 48, 211-219.

Williams, A. M., \& Burwitz, L. (1993). Advance cue utilization in soccer. In T. Reilly, J. Clarys, \& A. Stibbe (Eds.), Science and football II (pp. 239-244). London: E. \& F. N. Spon.

Williams, A. M., Davids, K., \& BuRwitz, L. (1995a). Visual search strategy in 3 vs. 3 and 1 vs. 1 situations in soccer. Journal of Sport \& Exercise Psychology, 17, S109.

Williams, A. M., Davids, K., \& Burwitz, L. (1995b). Visual search strategy and selective attention in 3 vs. 3 and 1 vs. 1 situations in soccer. Journal of Sport \& Exercise Psychology, 17, S109.
Williams, A. M., Davids, K., Burwitz, L., \& Williams, J. G. (1992). Perception and action in sport. Journal of Human Movement Studies, 22, 147-205.

Williams, A. M., Davids, K., Burwitz, L. \& Williams, J. G. (1993). Visual search and sports performance. Australian Journal of Science \& Medicine in Sport, 22, 55-65.

Williams, A. M., Davids, K., Burwitz, L., \& Williams, J. G. (1994). Visual search strategies in expert and novice soccer players. Research Quarterly for Sport \& Exercise Science, 65, 2, 127-135.

YARBUS, A. L. (1967). Eye movements and vision. New York: Plenum.

ZEGERS, D. H. A. (1986, Autumn). Some experiments with the NAC V eyemark recorder during agricultural selection tasks. Agricultural Engineer, pp. 99-102.

\section{NOTES}

1. Available from Applied Science Laboratories, 335 Bear Hill Road, Waltham, MA 02154

2. An independent-samples $t$ test revealed statistically significant differences in choice reaction time between the verbal report $(M=$ $347.70 \mathrm{msec}, S D=55.51 \mathrm{msec})$ and eye movement $(M=460.94 \mathrm{msec}$, $S D=90.91 \mathrm{msec})$ conditions $[t(40)=27.86, p<.01]$. However, an independent-samples $t$ test showed no significant differences in choice reaction time between the experienced ( $M=412.42 \mathrm{msec}, S D=$ $100.22 \mathrm{msec})$ and less experienced $(M=396.22 \mathrm{msec}, S D=81.79 \mathrm{msec})$ groups $[t(40)=0.56, p>.05]$.

3. Pearson product-moment correlation revealed a statistically significant correlation between the RT and VRE dependent variables $[r(40)=-.56, p<.001]$.

4. The relevance of these findings to the expertise literature is discussed elsewhere (see Williams et al., 1994).

5. This measure is actually total response time (TRT) and not choice reaction time (CRT). That is, it is a combination of decision time (DT) and movement time (MT). TRT was used as a covariate because it accounted for between-group differences in both DT and MT. Nevertheless, a one-way MANOVA was undertaken to check whether there were any differences in performance on the CRT task across groups. In this analysis, group (experienced/less experienced) was the betweensubjects factor, and DT, MT, and TRT were the dependent measures. The results showed a significant difference between groups [Wilks's lambda $=0.34,[F(3,20)=12.65, p<.0001]$. Univariate analysis showed that the less experienced subjects had faster DT $[F(1,22)=$ $6.05, p<.05]$ and TRT $[F(1,22)=8.35, p<.001]$. There were no differences in MT $[F(1,22)=0.72, p>.05]$.

6. Pearson product-moment correlation revealed a statistically significant correlation between the TRT and VRE dependent variables $[r(48)=-.73, p<.0001]$.

7. In this experiment, the subjects were required to respond by physically moving in the appropriate direction rather than verbally re sponding as in Experiment 1. It is acknowledged that this may have had a confounding effect when attempting to compare the effects of concurrent verbalization on performance reactivity across the two experiments. However, recent research has suggested that there may be different routes in the visual cortex for verbal and motor outputs. It appears that visual input is characterized by a more rapid direct route to the motor system (see Treisilian, 1995). Any confounding effect is therefore likely to be in the opposite direction to that observed in these experiments. That is, theoretically, Experiment 1, which involved visual input/verbal output, should have resulted in greater reactivity than the visual input/motor output condition employed in Experiment 2.

8 . The relevance of these findings to the expertise literature is discussed elsewhere (see Williams et al., 1995a).

(Manuscript received April 10, 1995 revision accepted for publication January 3,1996 .) 